\title{
Breast cancer incidence, stage, treatment and survival in ethnic groups in South East England
}

\author{
RH Jack*,', EA Davies' and H Møller' \\ 'King's College London, Thames Cancer Registry, Ist Floor Capital House, 42 Weston Street, London SEI 3QD, UK
}

Studies from the US have shown variations in breast cancer incidence, stage distribution, treatment and survival between ethnic groups. Data on 35631 women diagnosed with breast cancer in South East England between 1998 and 2003 with self-assigned ethnicity information available were analysed. Results are reported for White, Indian, Pakistani, Bangladeshi, Black Caribbean, Black African and Chinese women. Age-standardised breast cancer incidence rate ratios, patterns of stage of disease at diagnosis, treatment, overall and breast cancer-specific survival were examined. All ethnic groups studied had lower age-standardised breast cancer incidence rates than White women, with Bangladeshi women having the lowest rate ratio (0.23, 95\% Cl: 0.20-0.26). White women were the most likely to have a stage recorded at diagnosis (adjusted proportion 75\%), and least likely to be diagnosed with metastatic disease (7\%). Black African women were the least likely to have a record of cancer surgery (63\%) or hormone therapy (32\%), and most likely to receive chemotherapy (38\%). After fully adjusting for age, socioeconomic deprivation, stage of disease and treatment received, there was no significant variation in breast cancer-specific survival. However, Black African women had significantly worse overall survival (hazard ratio 1.24, $P=0.025$ ). These findings suggest that a strategy of earlier detection should be pursued in Black and South Asian women.

British Journal of Cancer (2009) I 00, 545-550. doi:10.1038/sj.bjc.6604852 www.bjcancer.com

Published online 6 January 2009

(c) 2009 Cancer Research UK

Keywords: ethnicity; breast cancer; incidence; stage; treatment; survival

Data from the United States show a lower breast cancer incidence in Black than White women, (Ward et al, 2004; Smigal et al, 2006) even after adjusting for socioeconomic status (Chlebowski et al, 2005). Using country of birth and names to assign ethnicity, a significantly lower incidence has been found in South Asian than in non-South Asian women (Winter et al, 1999; Smith et al, 2003), and in women born in the 'Caribbean Commonwealth' and South Asia than in the general female population in England and Wales (Harding and Rosato, 1999).

Many comparisons of stage of disease at diagnosis and treatment of cancer patients by ethnic group have been made in the United States. Whereas Black (Li et al, 2003; Morris et al, 2007) and Indian/Pakistani women (Li et al, 2003) were more likely to have advanced stage disease than White women, Hahn et al (2007) found no association between ethnicity and advanced stage after adjustment for several factors. Black women in the US also appear less likely to receive systemic chemotherapy and hormone therapy (Naeim et al, 2006) and more likely to receive inappropriate treatment compared to national guidelines ( $\mathrm{Li}$ et al, 2003) than White women. US studies have shown that Black women have a lower survival (Grann et al, 2006; Smigal et al, 2006), Chinese women have a better survival, and there is no difference between Indian/Pakistani women's survival compared with White women (Li et al, 2003).

*Correspondence: Ms RH Jack; E-mail: ruth.jack@kcl.ac.uk Revised 24 November 2008; accepted 2 December 2008; published online 6 January 2009
The area of South East England covered by the Thames Cancer Registry (TCR) is the most ethnically diverse in England, including groups who are from low incidence areas of the world. Hospital Episode Statistics (HES) data are available for all inpatient admissions to English NHS hospitals and include self-assigned ethnicity, which is more complete than that in TCR (Jack et al, 2006). This study used all available ethnicity data to describe patterns of breast cancer incidence, stage, treatment and survival in different ethnic groups in South East England.

\section{MATERIALS AND METHODS}

In the United Kingdom, cancer registries record the occurrence of cancer in their resident populations. In the area covered by the TCR, registration is initiated by clinical and pathological information received from hospitals and by information about deaths provided by the National Health Service Central Register through the Office for National Statistics. Trained data collection officers then extract further information on demographic details, disease stage and treatment in the first 6 months after diagnosis from the medical records. TCR uses a simple four-level staging system, using information in the patients' notes. This allows solid tumours to be assigned to categories based on whether the disease is local, has direct extension beyond the organ of origin, has regional lymph node involvement, or has metastasised. Data are quality assured as they are added to the central database. Hospital Episode Statistics data come from hospital Patient Administration Systems and include patient, clinical, administrative and geographical 
details. Self-assigned ethnicity was introduced to the HES data in April 1995 using the codes from the England and Wales 1991 Census. In April 2001 the new 2001 Census ethnic codes were brought in, although the 1991 codes were still accepted until March 2003.

Records on 55710 women diagnosed with breast cancer (ICD-10 code C50) between 1998 and 2003 were extracted from the TCR database. Hospital Episode Statistics data on all patients residing in South East England with cancer (or suspected cancer) admitted to NHS hospitals between April 1997 and March 2004 were obtained. These patient records were matched to the TCR records using either NHS number, or sex, date of birth and postcode. The ethnicity code was extracted from HES and added to the TCR record. If there was no match, and an ethnic code was available from TCR records, this was used.

Ethnic groups were classified into the following categories: White, Mixed, Indian, Pakistani, Bangladeshi, Asian Other, Black Caribbean, Black African, Black Other, Chinese, Other or Not Known. Although analyses were performed using all available information, results are only presented for the seven groups that are easily combined from the 1991 and 2001 Censuses: White, Indian, Pakistani, Bangladeshi, Black Caribbean, Black African and Chinese. Socioeconomic deprivation was measured using the income domain of the Index of Multiple Deprivation 2000 (Department of the Environment, Transport and the Regions, 2000), divided into quintiles across England and Wales and assigned to records using postcode of residence at diagnosis.

It was not possible to determine an ethnic code for all patients. As there is no 'ethnicity not known' group in the population data based on the census, the absolute values of computed agestandardised incidence rates by ethnic group are too low due to the exclusion of the patients without a valid ethnic code. To address this, age-standardised rate ratios were calculated, using Census 2001 data as the population denominator, with White women as the baseline group. Confidence intervals were calculated using the method described in Boyle and Parkin (1991). These were also calculated separately for three truncated age groups: under 50, $50-64$ and 65 years and older.

Patients who are registered by death certificate only have limited information recorded in the TCR database. These patients are included in the incidence analyses, but excluded from analyses on stage, treatment and mortality. There were 2117 (3.8\%) cases registered by death certificate only in this dataset. Logistic regression was performed to analyse the availability of the patients' stage at diagnosis. If stage was recorded, logistic regression was again used to determine whether the patient was diagnosed with metastatic disease. Results from both analyses were transformed to generate proportions adjusted for age and socioeconomic deprivation. The proportions of each ethnic group receiving non-investigative cancer surgery, radiotherapy, chemotherapy, and hormone therapy within the first 6 months of diagnosis were calculated. Using logistic regression these proportions were adjusted for age at diagnosis, socioeconomic deprivation and stage at diagnosis.

Survival was analysed using Cox regression, with patients followed up until 31 December 2006. Deaths from all causes and from breast cancer were analysed separately. A death was considered to have been due to breast cancer if the ICD-10 code C50, ICD-9 code 174 or the word 'breast' were included in Part 1 of the death certificate. The estimated hazard ratios (HR) were sequentially adjusted for age, socioeconomic deprivation, stage at diagnosis and treatment received.

\section{RESULTS}

Ethnicity information was available for 35631 (64\%) of the 55710 records of women diagnosed with breast cancer extracted from the database. Excluding cases registered by death certificate only, 34998 (65\%) of 53593 patients had a known ethnicity. Incidence results are reported for the seven ethnic groups, which make up $60 \%(33633)$ of the total cases. In all 33024 (62\%) cases had complete registration information rather than incomplete information from a death certificate only and the results for stage, treatment and survival analyses are shown for this group.

White women had the highest age-standardised breast cancer incidence rates. Incidence rate ratios were calculated with White women as the baseline group, and were all significantly below one: Indian (0.68; 95\% CI, 0.64-0.73), Pakistani (0.59; 95\% CI, $0.51-0.69)$, Bangladeshi (0.23; 95\% CI, 0.20-0.26), Black Caribbean (0.80; 95\% CI, 0.74-0.86), Black African $(0.66 ; 95 \%$ CI, 0.59-0.74) and Chinese (0.54; 95\% CI, 0.47-0.63).

Figure 1 shows age-standardised incidence rate ratios calculated separately for different age groups. Younger women of different ethnic groups had incidence rates more similar to White women of the same age. This pattern was evident in Bangladeshi, Black Caribbean, Black African and Chinese women, but was less clear for Pakistani women. There was no difference in the incidence rate ratios for the different age groups of Indian women.

Results of the analyses of stage, adjusted for age and socioeconomic deprivation, are shown in Table 1 . White women were most likely to have a stage recorded at diagnosis (75\%), and Bangladeshi women were least likely (55\%). After excluding patients with stage not known, Pakistani women were most likely to be diagnosed with metastatic disease $(17 \%)$ and White women the least likely (7\%). White women were less likely to be diagnosed with metastatic disease than all other groups, although the difference was not significant for Bangladeshi and Chinese women due to small numbers.

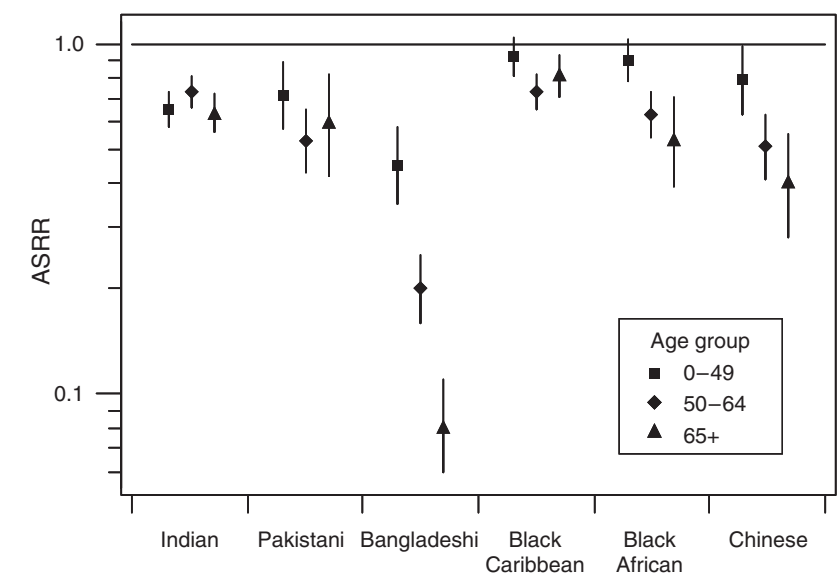

Figure I Age-standardised breast cancer incidence rate ratios (ASRR) and $95 \%$ confidence intervals by age group with White women as the baseline group.

Table I Percentage of breast cancer patients having a stage recorded at diagnosis, and of staged patients, those with metastatic disease at diagnosis by ethnic group, adjusted for age and socioeconomic deprivation

\begin{tabular}{|c|c|c|c|c|c|c|c|}
\hline & \multirow[b]{2}{*}{ Total } & \multicolumn{3}{|c|}{ Staged } & \multicolumn{3}{|c|}{ Metastatic disease } \\
\hline & & $n$ & $\%$ & $P$-value & $n$ & $\%$ & $P$-value \\
\hline White & 31109 & $2327 \mid$ & 75 & & 1743 & 7 & \\
\hline Indian & 639 & 446 & 67 & $<0.001$ & 43 & 11 & 0.012 \\
\hline Pakistani & 121 & 86 & 69 & 0.128 & 13 & 17 & 0.003 \\
\hline Bangladeshi & 42 & 25 & 55 & 0.007 & 3 & 13 & 0.297 \\
\hline Black Caribbean & 652 & 493 & 73 & 0.270 & 57 & 11 & 0.003 \\
\hline Black African & 344 & 253 & 71 & 0.082 & 36 & 15 & $<0.001$ \\
\hline Chinese & 117 & 85 & 71 & 0.388 & 7 & 10 & 0.435 \\
\hline
\end{tabular}


Table 2 shows the proportions of each ethnic group receiving different treatments, adjusted for age, socioeconomic deprivation and stage. The largest differences were seen between White and Black African women. Black African women were less likely to have a record of cancer surgery (63 vs 72\%, $P=0.003)$ and hormone therapy ( 32 vs 54\%, $P<0.001$ ), and more likely to receive chemotherapy (38vs 29\%, $P=0.001$ ) than White women. Pakistani women were significantly less likely to receive radiotherapy (27 vs $36 \%, P=0.043$ ) and hormone therapy ( $41 v s 54 \%, P=0.014$ ) than White women, whereas Black Caribbean women were less likely to receive hormone therapy (39 vs 54\%, $P<0.001$ ). Several other associations were of borderline significance, or not significant because of the small numbers. Bangladeshi $(41 \%, P=0.179)$, Chinese $(47 \%, P=0.191)$ and Indian $(50 \%, P=0.074)$ women were less likely to receive hormone therapy than White women (54\%); Chinese (28 vs 36\%, $P=0.088$ ) and Black Caribbean (32 vs $36 \%$, $P=0.078$ ) were less likely to receive radiotherapy, and Chinese women were also more likely to receive surgery (79 vs $72 \%$, $P=0.148)$.

The patterns of survival using death from any cause are shown in Table 3, sequentially adjusted for age, socioeconomic deprivation, stage and treatment. Chinese women had a lower risk of dying than White women, which was largely unaffected by adjustment (fully adjusted $\mathrm{HR}=0.66, P=0.088$ ). All other ethnic groups had higher risks of dying compared with White women after adjustment for age only. However, only Black African women's risk of dying remained high after additional adjustment for socioeconomic deprivation and stage $(\mathrm{HR}=1.42, P<0.001)$, and although attenuated, was still high after further adjustment for treatment $(\mathrm{HR}=1.24, P=0.025)$. Women living in the most socioeconomically deprived area had the worst overall survival (HR 1.25, $P<0.001$ ).

Results for breast cancer-specific survival, again adjusted for age, socioeconomic deprivation, stage and treatment, are shown in Table 4. There was a similar pattern to the overall survival: Chinese women had the lowest risk of dying from breast cancer (fully adjusted $\mathrm{HR}=0.63, P=0.089$ ) and Black African women had the highest (fully adjusted $\mathrm{HR}=1.09, P=0.412$ ). The worse breast cancer-specific survival in the other groups was attenuated by adjustment, and no significant association was found after adjusting for age, socioeconomic deprivation, stage and treatment.

\section{DISCUSSION}

This study found variations in breast cancer incidence, stage at diagnosis and treatment received between different ethnic groups

Table 2 Percentage of breast cancer patients receiving different treatments by ethnic group, adjusted for age, socioeconomic deprivation and stage

\begin{tabular}{|c|c|c|c|c|c|c|c|c|c|c|c|c|c|}
\hline & \multirow[b]{2}{*}{ Total } & \multicolumn{3}{|c|}{ Cancer surgery } & \multicolumn{3}{|c|}{ Radiotherapy } & \multicolumn{3}{|c|}{ Chemotherapy } & \multicolumn{3}{|c|}{ Hormone therapy } \\
\hline & & $n$ & $\%$ & $P$-value & $n$ & $\%$ & $P$-value & $n$ & $\%$ & $P$-value & $n$ & $\%$ & $P$-value \\
\hline White & 31109 & 22421 & 72 & & 1 | 060 & 36 & & 9006 & 29 & & 16678 & 54 & \\
\hline Indian & 639 & 469 & 70 & 0.398 & 231 & 35 & 0.955 & 229 & 26 & 0.185 & 275 & 50 & 0.074 \\
\hline Pakistani & 121 & 91 & 75 & 0.584 & 32 & 27 & 0.043 & 51 & 30 & 0.749 & 40 & 41 & 0.014 \\
\hline Bangladeshi & 42 & 29 & 69 & 0.706 & 13 & 36 & 0.949 & 19 & 27 & 0.770 & 11 & 41 & 0.179 \\
\hline Black Caribbean & 652 & 492 & 73 & 0.712 & 199 & 32 & 0.078 & 276 & 32 & 0.156 & 203 & 39 & $<0.001$ \\
\hline Black African & 344 & 234 & 63 & 0.003 & 100 & 33 & 0.390 & 205 & 38 & 0.001 & 70 & 32 & $<0.001$ \\
\hline Chinese & 117 & 95 & 79 & 0.148 & 31 & 28 & 0.088 & 58 & 30 & 0.834 & 43 & 47 & 0.191 \\
\hline
\end{tabular}

Table 3 Hazard ratios for all cause mortality in breast cancer patients diagnosed 1998-2003

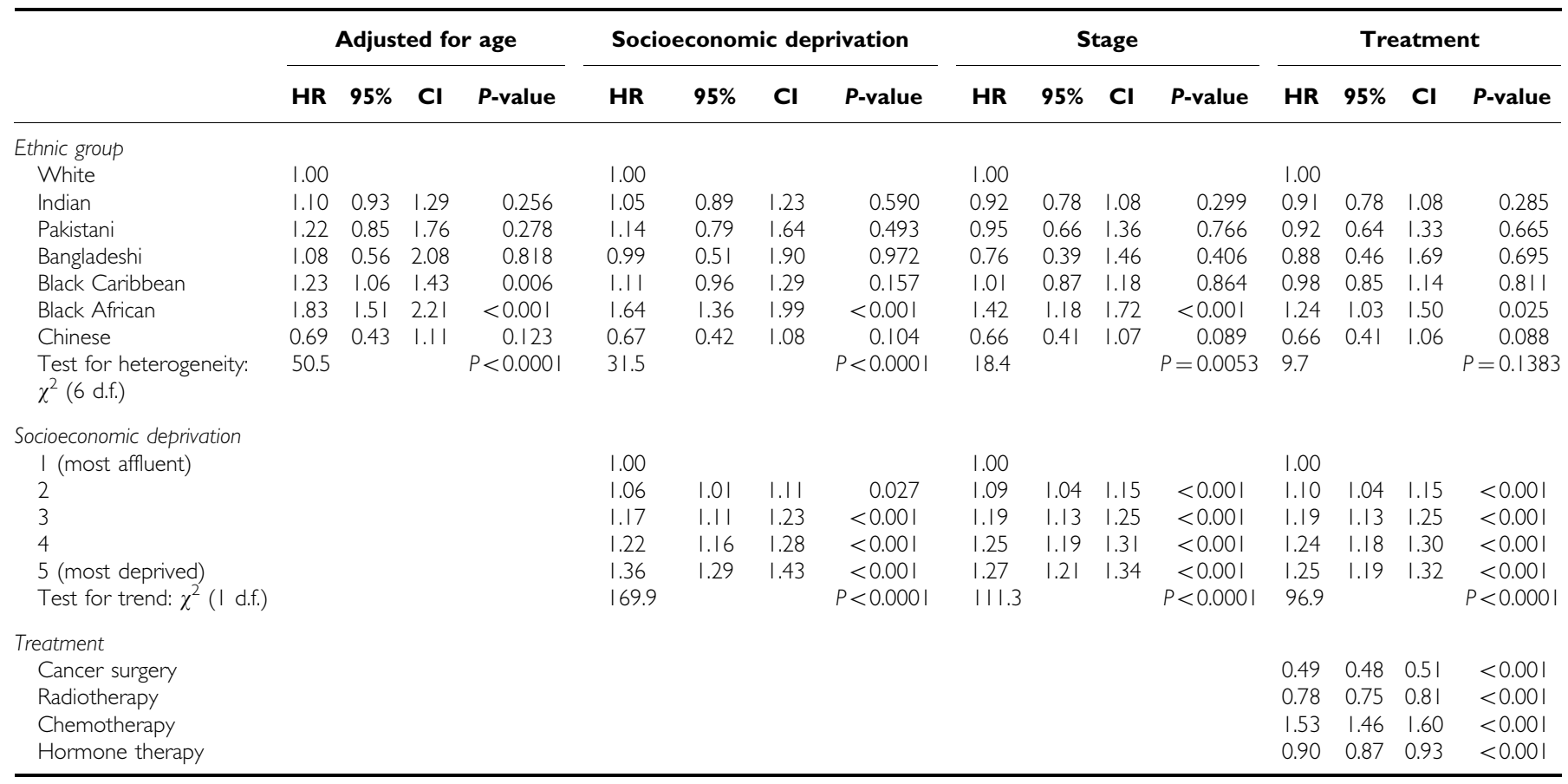


Table 4 Hazard ratios for breast cancer mortality (coded cause Ia, Ib or Ic mentioning 'C50' or 'I 74' or text cause Ia, Ib or Ic mentioning 'breast') in breast cancer patients diagnosed 1998-2003

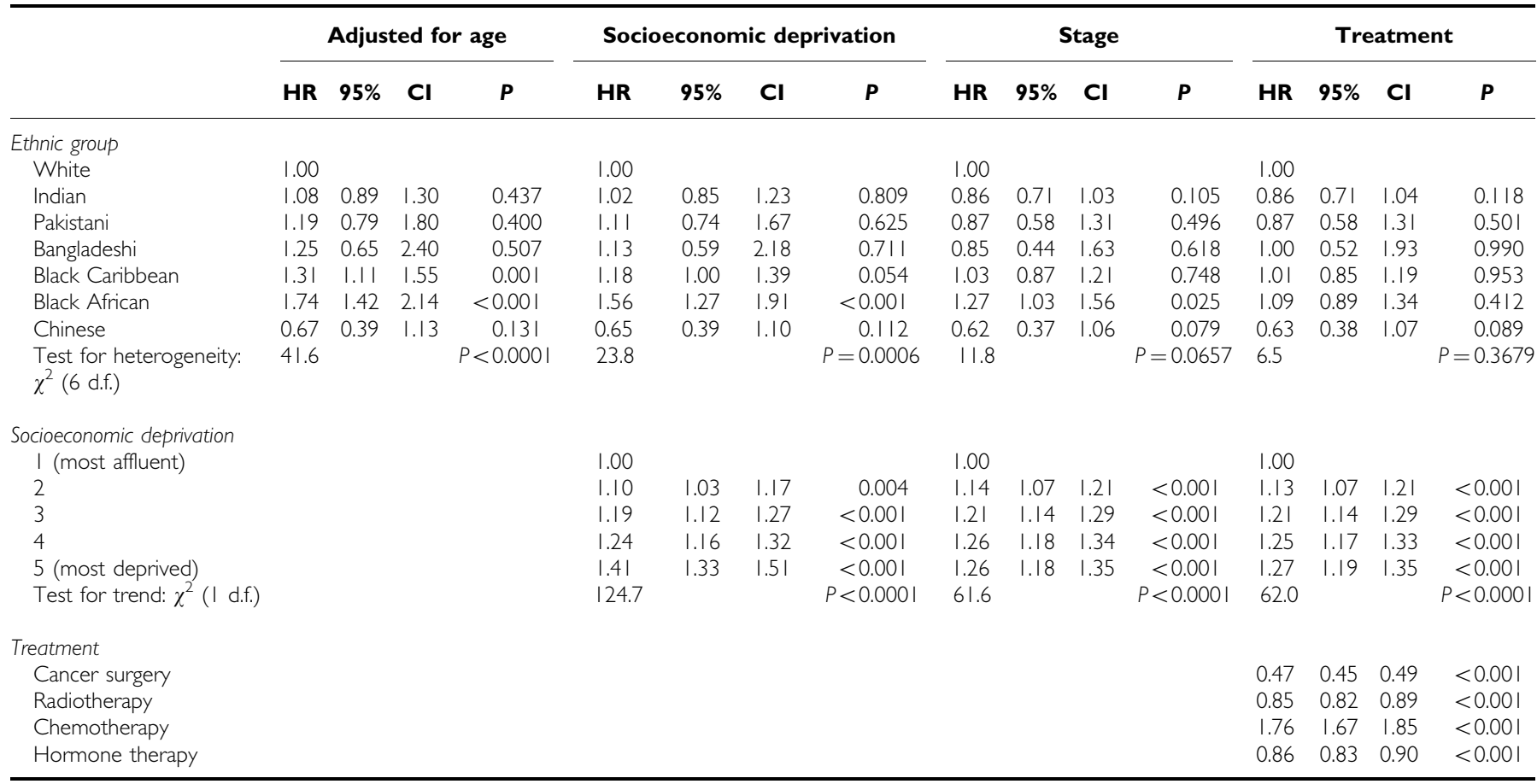

in South East England. White women had the highest agestandardised incidence rates and Bangladeshi women the lowest. The rates in younger women from different ethnic groups appeared to be converging. White women were most likely to have a stage recorded at diagnosis and least likely to present with advanced stage disease. There was significant variation in the treatment received by different ethnic groups, but these differences were generally consistent with differences in the proportion of patients with advanced disease.

There was less variation in survival between ethnic groups. Chinese women had the best overall and breast cancer-specific survival. Black African women had the worst overall survival, but the effect was not as strong for breast cancer-specific survival. Variation among other ethnic groups was explained by adjustment for other variables. Although adjustment for stage and treatment explained the variation in breast cancer-specific survival, it did not explain the worse survival for women living in more deprived areas.

This study replicates previous findings that White women have higher incidence rates than Black women in the United States (Ward et al, 2004; Chlebowski et al, 2005; Smigal et al, 2006) and the United Kingdom (Harding and Rosato, 1999). It also confirms that South Asian women have lower incidence rates than White women (Harding and Rosato, 1999; Winter et al, 1999; Smith et al, 2003), and is able to verify this separately for Indian, Pakistani and Bangladeshi women.

Screening detects cancers which are asymptomatic and may not have otherwise been diagnosed or recorded. There is evidence that in the United Kingdom screening uptake is lower in women who live in more deprived areas (Maheswaran et al, 2006) and that White British people are less likely to live in the most deprived areas of England (Tinsley and Jacobs, 2006). The analyses of incidence in this study did not take deprivation into account and therefore the higher incidence rates in White women could be partly due to more screen-detected disease. Screening will also affect the stage of disease, as it will detect less advanced tumours before they become symptomatic. However, even after adjusting for socioeconomic deprivation, the results of this study are consistent with previous findings of more advanced disease at diagnosis in Indian, Pakistani and Black women (Li et al, 2003; Morris et al, 2007). Hahn et al (2007) found no difference in disease stage at diagnosis between Black and White women after adjustment for several factors, including age, level of education, insurance status, poverty, method of detection and tumour characteristics. It is possible that some of these factors would explain the variation found in the present study.

In the United States, Black women are less likely to receive chemotherapy and hormone therapy (Naeim et al, 2006). In our study, Black Caribbean and Black African women were less likely to receive hormone therapy, but the latter were more likely to receive chemotherapy. This pattern of treatment in Black African women may reflect differences in stage and triple-negative disease (oestrogen receptor, progesterone receptor and human epidermal growth factor receptor 2 (HER2) negative). Studies in the United States have shown that Black women are more likely to have triplenegative breast cancer than White women (Bauer et al, 2007; Morris et al, 2007). This was also suggested by a study of patients below age 60 in a hospital population in London, although this result was not significant (Bowen et al, 2008). Triple-negative disease does not respond to hormone therapy, and chemotherapy may be the preferred treatment option for these cancers (Cleator et al, 2007).

US Black women had worse survival than White women, even after accounting for patient, tumour and geographical factors (Li et al, 2003; Grann et al, 2006). Chinese women had better survival than White women, and there was no significant difference between Indian/Pakistani and White women after adjusting for age, tumour characteristics and treatment (Li et al, 2003). An earlier study in the TCR area found women with South Asian names had better relative survival than non-South Asian women (dos Santos Silva et al, 2003). In a London hospital population Black women with smaller tumours had significantly worse overall survival than White women with similar sized tumours, adjusting for age and socioeconomic deprivation (Bowen et al 2008). In our study, Black African women had significantly 
worse overall survival after adjustment for age, socioeconomic deprivation, stage at diagnosis and treatment, and Chinese women had better survival, although not significantly so due to small numbers. No significant variation in survival was found in Indian, Pakistani or Bangladeshi women.

A significant limitation of this study is that ethnicity information was not available for a large proportion (36\%) of the patients. This could potentially bias the results if particular ethnic groups were over or underrepresented in the missing data. The computed incidence rate ratios assume that the proportion of missing values was the same in each of the different ethnic groups. All women diagnosed, including those with unknown ethnicity, were included in the analyses for stage, treatment and survival. Women with unknown ethnicity had a lower risk of dying from all causes (fully adjusted $\mathrm{HR}=0.89, P<0.001)$ and from breast cancer $(\mathrm{HR}=0.90$, $P<0.001)$ than White women. A sensitivity analysis was performed using the extreme assumption that all women of unknown ethnicity were White. The results for the other ethnic groups compared with this extended White group were very similar to the ones presented. Black African women had a significantly high fully adjusted HR when using all causes of death $(\mathrm{HR}=1.29, P=0.009)$, and although their breast cancer-specific survival was also high, it was not statistically significant $(\mathrm{HR}=1.13, P=0.252)$. All other ethnic groups had non-significant HRs compared with the new group of White women and women with unknown ethnicity. Improving ethnicity data collection is a key target for routinely collected datasets including hospital records, HES, and cancer registers.

Although this study was able to investigate ethnic groups at a more detailed level than most previous studies, there could still be important differences within these groups. For example, McCormack et al (2004) examined groups defined by place of birth (including area within country), first language and religion. The associations between these groups and risk of breast cancer lost their significance after adjustment for reproductive, socioeconomic, anthropometric and dietary factors. These and other factors such as comorbidity, which are not routinely recorded by cancer registries could contribute to the differences in treatment and survival. As the registry only collects information on treatment received up to 6 months after diagnosis, some treatment data are missing, although this is unlikely to vary by ethnic group.

The interpretation of survival differences between ethnic groups must recognise the likelihood of strong associations between socioeconomic factors, disease stage at diagnosis and treatment. In the analysis of breast cancer-specific survival, there was a gradual attenuation of the survival differences between ethnic groups as more variables were adjusted for. The adjustment for socioeconomic deprivation, disease stage and treatment all tended to work towards a reduction of the excess mortality in the South
Asian and Black groups. The most plausible interpretation is that survival differences are principally due to variation in stage at presentation, which in these data is manifest both through the recorded stage at diagnosis, but also independently through the recorded socioeconomic deprivation and the administered treatment. It seems very unlikely that there are biologically based differences between the ethnic groups, which confer strong survival differences, independently of stage and treatment. The only subanalysis that suggests this is the fully adjusted analysis of overall survival in Black African women where the HR persisted at a statistically significant value of 1.24 . However, this estimate was highly sensitive to adjustment for deprivation, stage and treatment, which leaves room for an effect of residual confounding. Further, the corresponding estimate in the breast cancer-specific analysis was much lower at 1.09. Therefore, the present data suggest that breast cancer services may usefully pursue a strategy of earlier detection with the aim of achieving a more favourable stage distribution at diagnosis. This should be a particular priority for the South Asian and Black groups analysed here.

To determine more robustly whether biological, behavioural or clinical factors are driving the observed differences, more information is needed on patient characteristics. The most important component is disease stage at diagnosis, as this will affect both the treatment received and survival. Improving the completeness of staging information as well as ethnicity information received by cancer registries will enhance future analyses. More advanced stage at diagnosis could reflect a more aggressive form of disease, later presentation because of a lack of awareness about symptoms or a mistrust of the health system, lower uptake of screening or delays in diagnosis. There is evidence that different ethnic groups have different ideas about causes of and candidacy for breast cancer (Pfeffer, 2004), which may affect attitudes towards accessing healthcare services. A better understanding of these beliefs would therefore be important to guide a strategy for earlier detection. Studies in the United States have also shown that Black women are more likely to have high risk tumour characteristics (Elledge et al, 1994) that are associated with earlier death from breast cancer (Anderson et al, 2005). Earlier detection is likely to be particularly important for these patients.

Although there are differences in recorded stage of disease at diagnosis and treatment between ethnic groups, it is encouraging that there is little variation in survival after adjustment for these factors, suggesting that some of the survival differences found in previous US studies (Grann et al, 2006; Smigal et al, 2006) may reflect the problems some groups have in accessing effective treatments in US healthcare systems. With better access to earlier detection, and the possibility of more aggressive treatment, survival could be improved for all women.

\section{REFERENCES}

Anderson WF, Jatoi I, Devesa SS (2005) Distinct breast cancer incidence and prognostic patterns in the NCI's SEER program: suggesting a possible link between etiology and outcome. Breast Cancer Res Treat 90: $127-137$

Bauer KR, Brown M, Cress RD, Parise CA, Caggiano V (2007) Descriptive analysis of estrogen receptor (ER)-negative, progesterone receptor (PR)negative, and HER2-negative invasive breast cancer, the so-called triplenegative phenotype: a population-based study from the California cancer Registry. Cancer 109: 1721 - 1728

Bowen RL, Duffy SW, Ryan DA, Hart IR, Jones JL (2008) Early onset of breast cancer in a group of British black women. Br J Cancer 98: 277-281

Boyle P, Parkin DM (1991) Statistical methods for registries. In Cancer registration principles and methods, Jensen OM, Parkin DM, MacLennan R, Muir CS, Skeet RG (eds), pp 126-158. International Agency for Research on Cancer: Lyon
Chlebowski RT, Chen Z, Anderson GL, Rohan T, Aragaki A, Lane D, Dolan NC, Paskett ED, McTiernan A, Hubbell FA, Adams-Campbell LL, Prentice R (2005) Ethnicity and breast cancer: factors influencing differences in incidence and outcome. J Natl Cancer Inst 97: 439-448

Cleator S, Heller W, Coombes RC (2007) Triple-negative breast cancer: therapeutic options. Lancet Oncol 8: 235-244

Department of the Environment, Transport and the Regions (2000) Indices of Deprivation 2000: Regeneration Research Summary No. 31. Stationery Office: London

dos Santos Silva I, Mangtani P, De Stavola BL, Bell J, Quinn M, Mayer D (2003) Survival from breast cancer among South Asian and non-South Asian women resident in South East England. Br J Cancer 89: 508-512 Elledge RM, Clark GM, Chamness GC, Osborne CK (1994) Tumor biologic factors and breast cancer prognosis among white, Hispanic, and black women in the United States. J Natl Cancer Inst 86: 705-712 
Grann V, Troxel AB, Zojwalla N, Hershman D, Glied SA, Jacobson JS (2006) Regional and racial disparities in breast cancer-specific mortality. Soc Sci Med 62: 337-347

Hahn KM, Bondy ML, Selvan M, Lund MJ, Liff JM, Flagg EW, Brinton LA, Porter P, Eley JW, Coates RJ (2007) Factors associated with advanced disease stage at diagnosis in a population-based study of patients with newly diagnosed breast cancer. Am J Epidemiol 166: $1035-1044$

Harding S, Rosato M (1999) Cancer incidence among first generation Scottish, Irish, West Indian and South Asian migrants living in England and Wales. Ethn Health 4: 83-92

Jack RH, Linklater KM, Hofman D, Fitzpatrick J, Møller H (2006) Ethnicity coding in a regional cancer registry and in Hospital Episode Statistics. BMC Public Health 6: 281

Li CI, Malone KE, Daling JR (2003) Differences in breast cancer stage, treatment, and survival by race and ethnicity. Arch Intern Med 163: $49-56$

Maheswaran R, Pearson T, Jordan H, Black D (2006) Socioeconomic deprivation, travel distance, location of service, and uptake of breast cancer screening in North Derbyshire, UK. J Epidemiol Community Health 60: 208-212

McCormack VA, Mangtani P, Bhakta D, McMichael AJ, dos Santos Silva I (2004) Heterogeneity of breast cancer risk within the South Asian female population in England: a population-based case-control study of firstgeneration migrants. $\mathrm{Br}$ J Cancer 90: 160-166
Morris GJ, Naidu S, Topham AK, Guiles F, Xu Y, McCue P, Schwartz GF, Park PK, Rosenberg AL, Brill K, Mitchell EP (2007) Differences in breast carcinoma characteristics in newly diagnosed African-American and Caucasian patients: a single-institution compilation compared with the National Cancer Institute's Surveillance, Epidemiology, and End Results database. Cancer 110: $876-884$

Naeim A, Hurria A, Leake B, Maly RC (2006) Do age and ethnicity predict breast cancer treatment received? A cross-sectional urban population based study. Breast cancer treatment: Age and ethnicity. Crit Rev Onco Hematol 59(3): 234-242

Pfeffer N (2004) Screening for breast cancer: candidacy and compliance. Soc Sci Med 58: $151-160$

Smigal C, Jemal A, Ward E, Cokkinides V, Smith R, Howe HL, Thun M (2006) Trends in breast cancer by race and ethnicity: update 2006. $C A$ Cancer J Clin 56: $168-183$

Smith LK, Botha JL, Benghiat A, Steward WP (2003) Latest trends in cancer incidence among UK South Asians in Leicester. Br J Cancer 89: 70-73

Tinsley J, Jacobs M (2006) Deprivation and Ethnicity in England: A Regional Perspective. Regional Trends 39: 19-28

Ward E, Jemal A, Cokkinides V, Singh GK, Cardinez C, Ghafoor A, Thun M (2004) Cancer disparities by race/ethnicity and socioeconomic status. CA Cancer J Clin 54: 78-93

Winter H, Cheng KK, Cummins C, Maric R, Silcocks P, Varghese C (1999) Cancer incidence in the south Asian population of England (1990-92) Br J Cancer 79: 645-654 